\title{
Thermal Decomposition and Kinetic Analysis of a Mil-88a Metal-organic Framework
}

\author{
M. G. Moustafa ( $\nabla$ mgmoustafa@azhar.edu.eg) \\ Al-Azhar University https://orcid.org/0000-0001-9304-4288 \\ Abdelaziz M. Aboraia \\ Southern Federal University \\ F. Elmasry \\ Jouf University \\ Vera V. Butova \\ Southern Federal University \\ Alexander Soldatov \\ Southern Federal University
}

\section{Research Article}

Keywords: MIL-88A metal-organic frameworks, Thermal decomposition, Kinetics analysis, Energy storage applications

Posted Date: May 10th, 2021

DOl: https://doi.org/10.21203/rs.3.rs-499655/v1

License: (c) (i) This work is licensed under a Creative Commons Attribution 4.0 International License.

Read Full License 


\title{
Thermal decomposition and kinetic analysis of a MIL-88A metal-organic framework
}

\author{
M. G. Moustafa ${ }^{a, *}$, Abdelaziz M. Aboraia ${ }^{b}$, F. Elmasry ${ }^{c}$, Vera V. Butova ${ }^{b, d}$, and \\ Alexander Soldatov ${ }^{b}$ \\ a Physics Department, College of Science and Arts, Jouf University, Qurayat, Saudi Arabia \\ ${ }^{\mathrm{b}}$ The Smart Materials Research Institute, Southern Federal University, Sladkova 178/24, 344090, \\ Rostov-on-Don, Russia. \\ ${ }^{\mathrm{c}}$ Physics Department, College of Science and Arts, Jouf University, Tabrjal, Saudi Arabia \\ ${ }^{\mathrm{d}}$ Federal Research Center of the Southern Scientific Center of the Russian Academy of Sciences, \\ 344006, Rostov-on-Don, The Russian Federation.
}

\begin{abstract}
The thermal decomposition of the MIL-88A metal-organic frameworks (MOFs) is studied, for the first time, at various heating rates under air and Nitrogen. The precursors of a MIL-88A material revealed water evaporation and remaining solvent molecules in the framework structure at low-temperatures range while at the hightemperatures range, the organic skeleton collapse; in the meantime, $\mathrm{Fe}_{0.94} \mathrm{O}$ and the shell of graphitized carbon are formed. Thermogravimetric analysis (TGA) curves of MIL-88A moved slowly to the high-temperatures region with increasing heating rates. Additionally, Differential Scanning Calorimetry (DSC) curves of MIL-88A provided a change in the peak intensity, indicating the difference in the sample weight loss. Xray diffraction (XRD) pattern and transmission electron microscopy (TEM) of the asprepared sample confirmed the existence of a single hexagonal MIL-88A without any impurities of other phases. However, XRD exhibited two phases $\mathrm{Fe}_{0.94} \mathrm{O}$ and $\mathrm{F}^{0}$, after annealing. TEM of the annealed sample also asserted the creation of a carbon layer on the surface. The activation energy and kinetic parameter of the MIL-88A material offered higher values as the exothermic peak increased. Interestingly, the thermal decomposition of the MIL-88A material is effective in investigating the temperature effect on the pyrolysis process that can benefit in energy storage applications.
\end{abstract}

Keywords: MIL-88A metal-organic frameworks; Thermal decomposition; Kinetics analysis; Energy storage applications.

*Corresponding author;

Email: mgmmohamed@ju.edu.sa,mgmoustafa@azhar.edu.eg (M. G. Moustafa) 


\section{Introduction}

Recently, the world is heading towards a green revolution to decrease pollution and carbon dioxide resulting from burning fossil fuels. Besides, our society increasingly needs high performance and safe energy storage devices [1]. Thus, the development of novel functional compounds with high performance is the critical step. One of these new functional materials is Metal-organic frameworks (MOFs). MOFs represent unique porous compounds and containing organic linkers and metal ions [2]. The definite assemblage of organic linkers and metal ions produces a solid structure that spreads in different directions with great holes [2,3], low densities, and ultrahigh surface areas. According to these properties, MOFs have revealed various applications in greenhouse gas capture [4], drug delivery [5], energy storage [6], and cancer therapy [7].

Several properties of MOFs have been studied, such as the structure, electronic, electrochemical properties. However, the thermal characteristic and kinetic parameters of MOFs are still scarce. Therefore, the thermal decomposition of MOFs deserves interest to concoct for potential applications according to cruel provisions. The thermal stability of MOFs dramatically depends on the strength of metal-ligand bonds and connectivity. Limited thermal stability is a major reason why MOFs are frequently outperformed by other compounds like MIL-88A in recent applications $[8,9]$. There are different types of MOF materials, such as UIO-66 (University of Oslo), MIL-88A (Materials Institute Lavoisier), and Zeolitic imidazolate frameworks (ZIFs). The MIL-88A material is a type of MOF that has some advantages, like higher porosity and great surface area than other MOFs. This study focuses on the MIL series, especially MIL-88A. The MIL series represents MOF structures that combine highly metal ions with carboxylate-based ligands, based on which many modified MOFs have been advanced by functional or extended bonds [10]. One of such series is a MIL-88A material with a three-dimensional supple framework based on $\mathrm{Fe}^{3+}$ octahedral edges of the oxocentra axis connected by fumarate dihines, producing interrelated cages and pores with open canals operating along the c-axis [11]. Such an organization offers high porosity and a large specific area, rendering it appropriate for several applications such as carbon dioxide separation and absorption [12]. The MIL88A material demonstrates a perfect content of coordinated unsaturated metal ions, 
which are the elementary reaction locations for host olefin molecules during $\pi$ complex, rendering it suitable to absorb gases such as ethylene [13].

There are several papers offered on MIL-88A material. Amaro-Gahete et al. [14] synthesized highly crystalline MIL-88A particles with excellent properties. The effect of the synthesis time and sonication generator on the morphology, crystallinity, surface area, and structure of these materials were investigated. Liu et al. [15] studied the structural and magnetic properties of $\mathrm{Fe}_{3} \mathrm{O}_{4} / \mathrm{MIL}-88 \mathrm{~A}$ composites. The adsorption behavior for Bromophenol Blue was estimated. Benítez et al. [16] synthesized MIL88A@S composites by fast ultrasonic-assisted probe technique. The structural and electrochemical properties of the MIL-88A@S composite were investigated. The MIL-88A@S compound exhibited excellent storage capacity at higher current density, signifying this composite is a promising electrode for Li-S batteries.

In general, reactions are conducted at high-temperature conditions for separation and adsorption utilizing MOFs materials [17], leading to the fact that thermal decomposition investigation of samples is required for materials application. Despite some papers that have been offered on the thermal decomposition of MOFs, the MIL88A material has not been studied in terms of thermal decomposition. Thus, the thermal characteristics of MIL-88A compounds deserve attention to investigate the thermal changes that occurred at different temperatures. Out of this point, this article sheds new light on the thermal decomposition of MIL-88A material. This work aims to prepare a MIL-88A material and to study the thermal decomposition of a MIL-88A material at various heating rates. Besides, the kinetic parameters of this material are calculated.

\section{Experimental procedures}

\subsection{Sample preparation}

Solution A: Fumaric acid (0.6964 g) in a conical flask was dissolved in $150 \mathrm{ml}$ of water at $70{ }^{\circ} \mathrm{C}$, and then cooled. Solution B: $\mathrm{FeCl}_{3} * 6 \mathrm{H}_{2} \mathrm{O} 1.6218 \mathrm{~g}$ (6 mmol) was dissolved in $10 \mathrm{ml}$ of water. Solution A was mixed with another one (solution B), and water was added up to a volume of $170 \mathrm{ml}$. The flask with the reaction mixture was located in a furnace at $70^{\circ} \mathrm{C}$ for $19 \mathrm{~h}$. The precipitate was separated through centrifugation, washed several times with Deionized water and methanol to remove any impurities of fumaric acid which did not contribute to the reactions, and then dehydrated at $60{ }^{\circ} \mathrm{C}$ overnight as demonstrated in schematic 1 . 


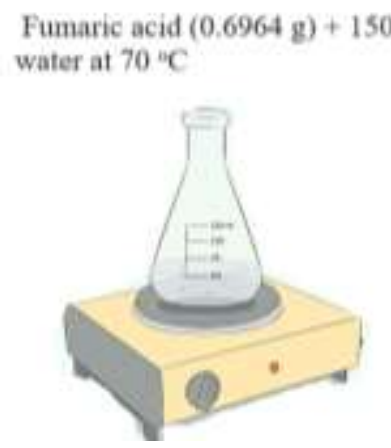

$\mathrm{FeCl}^{*} 6 \mathrm{H}_{2} \mathrm{O} 1.6218 \mathrm{~g}+10 \mathrm{ml}$ DI water

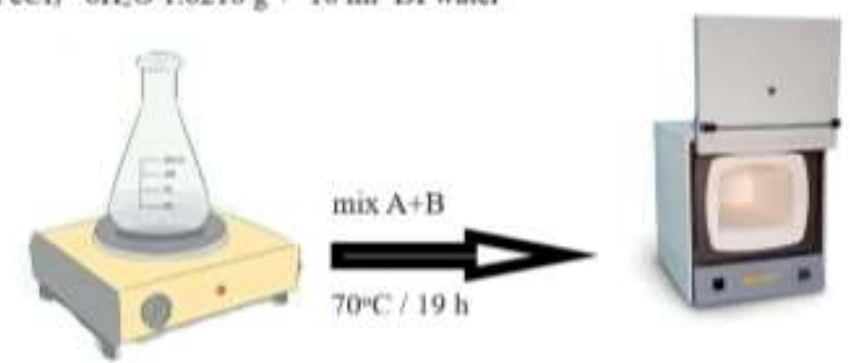

Schematic 1: The MIL-88A synthesizing method

\subsection{Sample characterization}

DSC and TGA tests were conducted using a thermobalance STA 449 F5 Jupiter. The powdered sample was contained in an alumina crucible and scanned at different heating rates $(\beta=5,10,15,20$, and $25 \mathrm{~K} / \mathrm{min})$. Linked data of pyrolysis process of the sample was obtained at a temperature ranging from 300 to $900{ }^{\circ} \mathrm{K}$, with accuracy $\pm 1 \mathrm{~K}$, under air and Nitrogen. The kinetics analysis was studied based on DSC data. XRD data was collected via X-ray diffractometer Bruker D2 Phaser $\left(\mathrm{CuK}_{\alpha}\right.$, $\lambda=1.5417 \AA$ ) and documented in the $2 \theta$ range of 5-80 with 0.0101 steps. Consequent refinement was done by the Rietveld route in the FullProf package, as observed in the Ref. [18]. The morphology analyses were described utilizing transmission electron microscopy (TEM) (Tecnai G2 Spirit Bio TWIN) operating at $120 \mathrm{kV}$.

\section{Results and discussion}

\subsection{TGA and DSC}

The pyrolysis process of the as-prepared MIL-88A metal-organic framework was tested by the thermogravimetric stability (TGA), which measured at a temperature ranging from 300 to $900{ }^{\circ} \mathrm{K}$ under Nitrogen and air. Figure 1(a) exhibits the TGA and DSC curves of the as-prepared MIL-88A metal-organic framework measured at a temperature ranging from 300 to $900{ }^{\circ} \mathrm{K}$ under air. It demonstrated that the precursors of MIL-88A suffered weight loss in stage A and additionally verified by exothermic of the process, as shown in Figure 1 (b), which may be referred to as water evaporation and remaining solvent molecules in the framework structure. As the temperature increased, the two steps of weight loss B and C stages were also detected due to the organic skeleton collapse; in the meantime, $\mathrm{Fe}_{0.94} \mathrm{O}$ and shell of graphitized carbon were formed [19]. This retention of carbon through the process of pyrolysis 
under air has been reported in other studies [20-22]. According to the further increasing temperature, the shells of carbon could be neglected from the yield product. The change in the peak intensity of DSC in Figure 1(b) refers to the difference in the sample weight loss. Based on TGA and DSC results (Fig. 1), the best temperature used to anneal the $\mathrm{Fe}_{0.94} \mathrm{O}$ coated by carbon shells at $723{ }^{\circ} \mathrm{K}$.

Figure 2(a,b) illustrates the TGA and DSC curves of the as-prepared MIL-88A metal-organic framework measured at a temperature ranging from 300 to $900{ }^{\circ} \mathrm{K}$ under $\mathrm{N}_{2}$ flow. There are principally three regions in a decomposition pattern, as shown in Figure 2(a). We noted a weight loss at around $373{ }^{\circ} \mathrm{K}$ due to water evaporation and the remaining solvent molecules at the first stage, also verified by the exothermicity of the process (Figure 2(b)). Furthermore, an organic ligand was gradually decomposed in a pair of stages, allowing the whole collapse of an organic structure at around $693^{\circ} \mathrm{K}[16,21]$. It is observed from Figures 1(a) and 2(a) that TGA curves move slowly to the high-temperatures region as the heating rates increased. This is due to the aggravation of the temperature gradient between the samples and the surrounding areas at high heating rates. It is also clear from Figures 1(b) and 2(b) that the exothermic peak $\left(T_{p}\right)$ in DSC curves moves to high temperatures as the heating rates increased, signifying the kinetic nature of the crystallization is present.

It is observed that the exothermic peaks obtained in DSC curves (Figures 1(b), and 2(b)) are broad, signifying the presence of more overlapped peaks. To separate overlapped peaks, the deconvolution process is applied using the Origin program with Gaussian-type function (Figure 3(a,b)). The deconvolution of overlapped exothermic peaks at heating rate $\beta=25 \mathrm{~K} / \mathrm{min}$ is offered in Figure $3(\mathrm{a}, \mathrm{b})$, as an example. The exothermic peaks overlapped may be due to phase separation that occurs upon the synthesizing process. The atmosphere of annealing affects the composition; hence in the case of annealing the materials under air, there are four stages from evaporating the water up to forming $\mathrm{Fe}_{0.94} \mathrm{O}$, and each stage is represented by a peak, as demonstrated in Figure 3(a). However, we noted three stages under $\mathrm{N}_{2}$ annealing from water evaporation up to forming carbon layer on the Fe, as shown in Figure 3(b).

\subsection{XRD and TEM}

Figure 4 shows the Rietveld refinement of the XRD pattern for MIL-88A. This refinement emphasized that the as-prepared composition is a single hexagonal MIL- 
88A with space group P-62c, lattice parameters $\mathrm{a}=13.29 \AA$, and $\mathrm{c}=12.57 \AA$, and there are no impurities by another crystalline phase. The observed data is a red line; the calculated pattern is a black line; the green line is Bragg reflection, and the blue line is the difference between the calculated and observed data. The XRD of the annealed sample is exhibited in Figure 4(b). It appears that all peaks are indexed to two phases $\mathrm{Fe}_{0.94} \mathrm{O}$ and $\mathrm{Fe}^{0}$, according to COD 2311020 and Pdf2-060696, respectively. To investigate the microstructure of MIL-88A materials after and before annealing, the measurements of TEM are utilized, as shown in Figure 5(a,b). The as-prepared MIL$88 \mathrm{~A}$ contains microcrystals with a mean width of $1 \mu \mathrm{m}$ and a smooth surface, as shown in Figure 5(a). However, after annealing under Ar at $723{ }^{\circ} \mathrm{K}$, the precursor of MIL-88A converted from solid to nano assembled bipyramid with layers from carbon formed on the surface Figure 5(b)[19].

\subsection{Kinetics analysis}

To examine the crystallization kinetics of MOF materials, kinetic's parameters, such as activation energy, can be determined. The most important kinetic parameter to understand the crystallization process of MOF materials is the activation energy. The isothermal and non-isothermal routes can be utilized. Most techniques are built on the Johnson-Mehl-Avrami (JMA) equation of isothermal transformation kinetics (MA'LEK 2000) [23]:

$$
x(t)=1-\exp \left(-k t^{n}\right)
$$

$x(t)$ represents the fraction of volume converted after time t, the $n$ represents Avrami kinetics exponent, which responsible for determining the rate of nucleation and the growth morphology), $k=$ constant of rate reaction. The temperature dependence is typically named from the Arrhenius equation [24]:

$$
k=k_{o} \exp \left(\frac{-E}{R T}\right)
$$

Where $E$ represents the activation energy for crystallisation reaction, $k_{o}$ represents the factor of frequency, the $R=$ gas constant. The crystallization of non-isothermal is described by the rate constant of heating. The activation energy of the MIL-88A can be determined by Kissinger's equation, which is obtained from Eq. (3), where $\beta$ is the 
heating rate, $T$ is the maximum peak temperature, $A$ is the frequency factor, and $R$ is the universal gas constant $(8.314 \mathrm{~J} / \mathrm{mol} \mathrm{K})[25,26,27]$.

$$
\ln \left(\frac{\beta}{T^{2}}\right)=\frac{-E}{R T}+\ln \left(\frac{R A}{E}\right)
$$

The temperature of max-peak for each exothermic reaction is affected by various heating rates like $5,10,15,20$, and $25^{\circ} \mathrm{K} / \mathrm{min}$, and it was revealed for it utilizing the

DSC non-isothermal technique. According to Eq.(3), plots of $\ln \left(\frac{T_{p}^{2}}{\beta}\right)$ versus $\frac{1000}{T_{p}}$ for the MIL-88A under air and Nitrogen are demonstrated in Figure $6(a, b)$. Based on the slope of lines, the activation energy for the MIL-88A under air and Nitrogen can be computed and exhibited in Table 1. It is observed that the activation energy and kinetic parameter of the MIL-88A under air and Nitrogen increase as the exothermic peak increased. This is due to the exothermic peak $\left(T_{p}\right)$ shifting to high temperatures as the heating rates increased. On the other hand, the kinetic parameter and the activation energy of the MIL-88A material provided this behavior due to the nature of the decomposition mechanism in the process of pyrolysis. Interestingly, the thermodynamics analysis of the MIL-88A material is an effective way to explore the temperature influence on the process of pyrolysis, which can benefit in several recent applications.

\section{Conclusion}

For the first time, the thermal decomposition of the MIL-88A MOF is explored at various heating rates under air and Nitrogen. The MIL-88A MOF was successfully synthesized. The first stage of the MIL-88A pyrolysis process is due to water evaporation and remaining solvent molecules in the framework structure. Besides, the second and third stages of the MIL-88A pyrolysis process are attributed to the organic skeleton collapse; in the meantime, $\mathrm{Fe}_{0.94} \mathrm{O}$ and the shell of graphitized carbon are formed. TGA curves of the MIL-88A sample shifted weakly to high-temperatures rang as the heating rates increased. Moreover, DSC curves of the MIL-88A sample offered a change in the peak intensity, signifying the difference in the sample weight loss. XRD pattern and TEM of the as-prepared sample affirmed the presence of a single hexagonal MIL-88A without any impurities of other phases. However, two phases $\mathrm{Fe}_{0.94} \mathrm{O}$ and $\mathrm{F}^{0}$ are present after annealing. TEM of the annealing sample 
confirmed the creation of a carbon layer on the surface. The activation energy and kinetic parameter of the MIL-88A increased with the increase of the exothermic peak. Remarkably, the thermal decomposition of the MIL-88A compound is an effective method for studying the temperature effect on the pyrolysis process that can be useful in energy storage applications.

\section{Declaration of Interest Statement}

The authors declare that they have no known competing financial interests or personal relationships that could have appeared to influence the work reported in this paper.

\section{Acknowledgments}

1- The authors extend their appreciation to the Deputyship for Research \& Innovation, Ministry of Education in Saudi Arabia for funding this work through the project number "1470020316".

2- The authors would like to extend their sincere appreciation to the central laboratory at Jouf University to support this study.

\section{References}

[1] M. Armand, J. M. Tarascon, Building Better Batteries, Nature 451(2008) 652 657

[2] J.L. Wang, C. Wang, W. Lin, Metal-organic frameworks for light-harvesting and photocatalysis, ACS Catal., 2 (2012), pp. 2630-2640, https://doi.org/10.1021/cs3005874

[3] A. Kirchon, L. Feng, H.F. Drake, E.A. Joseph, H.C. Zhou, From fundamentals to applications: a toolbox for robust and multifunctional MOF materials, Chem. Soc. Rev., 47 (2018), pp. 8611-8638. DOI: 10.1039/C8CS00688A

[4] R.W. Flaig, T.M. Osborn Popp, A.M. Fracaroli, E.A. Kapustin. M.J. Kalmutzki, R.M. Altamimi, F. Fathieh, J.A. Reimer, O.M. Yaghi, The chemistry of $\mathrm{CO}_{2}$ capture in an amine-functionalized metal-organic framework under dry and humid conditions, J. Am. Chem. Soc., 139 (2017), pp. 12125-12128. https://doi.org/10.1021/jacs.7b06382 
[5] M.X. Wu, Y.W. Yang, Metal-organic framework (MOF)-based drug/cargo delivery and cancer therapy, Adv. Mater., 29 (2017), p. 1606134.

[6]T. Wei, M. Zhang, P. Wu, Y.J. Tang, S.L. Li, F.C. Shen, X.L. Wang, X.P. Zhou, Y .Q. Lan, POM-based metal-organic framework/reduced graphene oxide nanocomposites with hybrid behavior of battery-supercapacitor for superior lithium storage, Nano Energy, 34 (2017), pp. 205-214. https://doi.org/10.1016/j.nanoen.2017.02.028

[7] G. Lan, K. Ni, Z. Xu, S.S. Veroneau, Y. Song, W. Lin, Nanoscale metal-organic framework overcomes hypoxia for photodynamic therapy primed cancer immunotherapy, J. Am. Chem. Soc., 140 (2018), pp. 5670-5673. https://doi.org/10.1021/jacs.8b01072

[8] S. Ma, X.S. Wang, D. Yuan, H.C. Zhou, A coordinatively linked Yb metal-organic framework demonstrates high thermal stability and uncommon gas-adsorption selectivity, Angew. Chem. Int. Ed. Engl., 47 (2008), pp. 4130-4133.

https://doi.org/10.1002/ange.200800312

[9] A.J. Howarth, Y. Liu, P. Li, Z. Li, T.C. Wang, J.T. Hupp, O.K. Farha, Chemical, thermal and mechanical stabilities of metal-organic frameworks, Nat. Rev. Mater., 1 (2016), pp. 1-15. https://doi.org/10.1038/natrevmats.2015.18

[10]S. Yuan, L. Feng, K. Wang, J. Pang, M. Bosch, C. Lollar, Y. Sun, J. Qin, X. Yang , P. Zhang, et al., Stable metal-organic frameworks: design, synthesis, and applications, Adv. Mater., 30 (2018), p. e1704303, https://doi.org/10.1002/adma.201704303

[11] C. Mellot-Draznieks, C. Serre, S. Surblé, N. Audebrand, G. Férey, Very large swelling in hybrid frameworks: a combined computational and powder diffraction study, J. Am. Chem. Soc., 127 (2005), pp. 16273-16278. https://doi.org/10.1021/ja054900x

[12]S. Wongsakulphasatch, W. Kiatkittipong, J. Saupsor, J. Chaiwiseshphol, P. Pironl erkgul, V. Parasuk, S. Assabumrungrat, Effect of Fe open metal site in metalorganic frameworks on post-combustion $\mathrm{CO}_{2}$ capture performance, Greenh. Gases Sci. Technol., 7 (2017), pp. 383-394. https://doi.org/10.1002/ghg.1662 [13]B. Li, Y. Zhang, R. Krishna, K. Yao, Y. Han, Z. Wu, D. Ma, Z. Shi, T. Pham, B. Space, J. Liu, P.K. Thallapally, J. Liu, M. Chrzanowski, S. Ma, Introduction of 
$\pi$-complexation into porous aromatic framework for highly selective adsorption of ethylene over ethane, J. Am. Chem. Soc., 136 (2014), pp. 8654-8660

[14] Juan Amaro-Gahete, Rafael Klee, Dolores Esquive, José Rafael Ruiz, César Jiménez-Sanchidrián, Francisco José Romero-Salguero, Fast ultrasound-assisted synthesis of highly crystalline MIL-88A particles and their application as ethylene adsorbents, Ultrasonics Sonochemistry, 50 (2019) 59-66.

https://doi.org/10.1016/j.ultsonch.2018.08.027

[15] Yi Liu, Yumin Huang, Aiping Xiao, Huajiao Qiu, Liangliang Liu, Preparation of Magnetic $\mathrm{Fe}_{3} \mathrm{O}_{4} / \mathrm{MIL}-88 \mathrm{ANanocomposite}$ and Its Adsorption Properties for Bromophenol Blue Dye in Aqueous Solution, Nanomaterials, 9 ( 2019) 51.

[16] Almudena Benítez, Juan Amaro-Gahete, Dolores Esquivel, Francisco José Romero-Salguero, Julián Morales, Álvaro Caballero, MIL-88A Metal-Organic Framework as a Stable Sulfur-Host Cathode for Long-Cycle Li-S Batteries, Nanomaterials, 10 (2020) 424.

[17] Yuan J, Zhu H, Sun J, Mao Y, Liu G, Jin W., Novel ZIF-300 mixed-matrix membranes for efficient $\mathrm{CO}_{2}$ capture, ACS Appl Mater Interfaces, 9 (2017) $38575-83$.

[18] V. V. Butova, O. A. Burachevskaya, I. V. Ozhogin, G. S. Borodkin, A. G. Starikov, S. Bordiga, A. Damin, K. P. Lillerud, and A. V. Soldatov, UiO-66 type MOFs with mixed-linkers-1,4-Benzenedicarboxylate and 1,4naphthalenedicarboxylate: Effect of the modulator and post-synthetic exchange, Microporous Mesoporous Mater., 305 (2020) 110324.

[19] Yang Wang, Xingmei Guo, Zhenkang Wang, Minfeng Lü, Bin Wu, Yue Wang, Chao Yan, Aihua Yuan and Hongxun Yang, Controlled pyrolysis of MIL-88A to $\mathrm{Fe}_{2} \mathrm{O}_{3} @ \mathrm{C}$ nanocomposites with varied morphologies and phases for advanced lithium storage, J. Mater. Chem. A, 5 (2017) 25562-25573.

[20] Li Feiyue, Cao Xinde, Zhao Ling, et al. Effects of mineral additives on biochar formation: Carbon retention, stability, and properties, Environmental Science and Technology, 48(19) (2014) 11211-11217.

[21] Yang, Y.; Shukla, P.; Wang, S.; Rudolph, V.; Chen, X.-M.; Zhu, Z. Significant improvement of surface area and $\mathrm{CO}_{2}$ adsorption of $\mathrm{Cu}-\mathrm{BTC}$ via solvent exchange activation. RSC Adv., 3 (2013) 17065-17072. 
[22] Li Wang, Yayun Zhang, Xia Li, Yingzhen Xie, Juan He, Jie Yu \& Yonghai Song, The MIL-88A-Derived $\mathrm{Fe}_{3} \mathrm{O}_{4}$-Carbon Hierarchical Nanocomposites for Electrochemical Sensing, 5 (2015) 14341.

[23] Ashutosh Goel, Essam R. Shaaban, Dilshat U. Tulyaganov, and Jose' M. F. Ferreira, Study of Crystallization Kinetics in Glasses along the Diopside-CaTschermak Join, J. Am. Ceram. Soc., 91 [8] (2008) 2690-2697.

[24] Y.B. Saddeek, E.R. Shaaban, F.M. Abdel-Rahim, K.H. Mahmoud, Thermal analysis and infrared study of $\mathrm{Nb}_{2} \mathrm{O}_{5}-\mathrm{TeO}_{2}$ glasses, 88 [5] (2008) 3059-3073.

[25] H. E. Kissinger, Reaction kinetics in differential thermal analysis, Anal. Chem., 29 (1957) 1702-1706.

[26] Mi R. Kim, Taehee Kim, Hyeong S. Rye, Wonjoo Lee, Hyeon-Gook Kim, Moon Il Kim, Bongkuk Seo, and Choong-Sun Lim, Zeolitic imidazolate framework promoters in one-pot epoxy-amine reaction, J. Mater. Sci., 55 [5] (2020) 20682076.

[27] E. R. Shaaban, M. Y. Hassaan, M. G. Moustafa, Ammar Qasem \& El Sayed Yousef, Sheet resistance-temperature dependence, thermal and electrical analysis of As40S60-xSex thin films, Applied Physics A, 126 (2020) 34. https://doi.org/10.1007/s00339-019-3217-1

Table 1 Activation energy and kinetic parameter of the MIL-88A pyrolysis process calculated by the non-isothermal method.

\begin{tabular}{cccc}
\hline Gas type & Peak & $\begin{array}{c}\text { Activation Energy } \\
(\mathbf{k J} / \mathbf{m o l})\end{array}$ & $\begin{array}{c}\text { Kinetic } \\
\text { parameter } \\
\left(\mathbf{s}^{-\mathbf{1}}\right)\end{array}$ \\
\hline \multirow{6}{*}{ Air } & Peak 1 & 244.739 & $4.506 \mathrm{E}+19$ \\
& Peak 2 & 264.859 & $3.406 \mathrm{E}+20$ \\
& Peak 3 & 300.928 & $1.065 \mathrm{E}+22$ \\
& Peak 4 & 346.9 & $6.426 \mathrm{E}+23$ \\
\hline \multirow{3}{*}{ Nitrogen } & Peak 1 & 246.428 & $5.357 \mathrm{E}+19$ \\
& Peak 2 & 269.395 & $5.317 \mathrm{E}+20$ \\
& Peak 3 & 405.774 & $8.392 \mathrm{E}+25$ \\
\hline
\end{tabular}


Figures
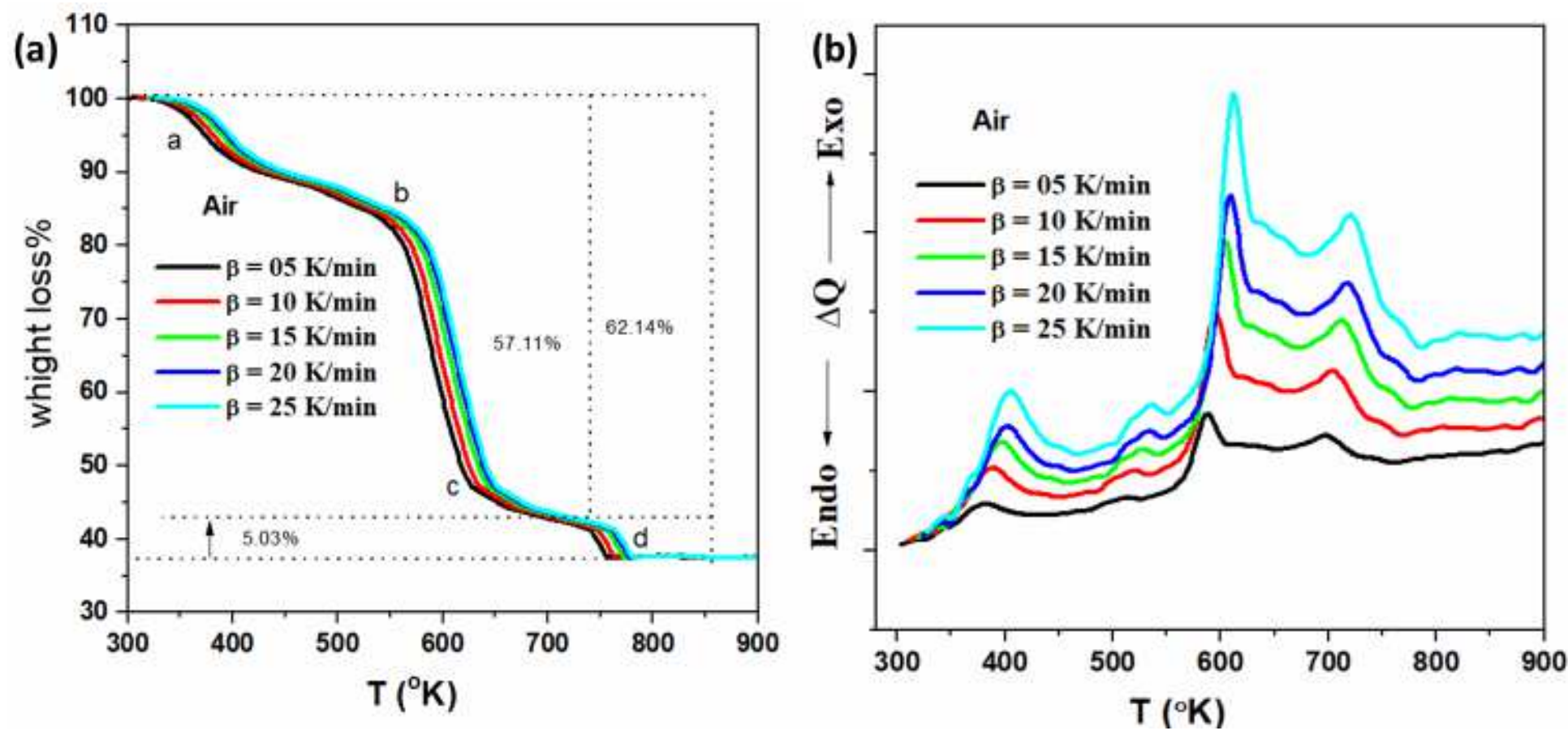

Figure 1

See the Supplemental Files section for the complete figure caption
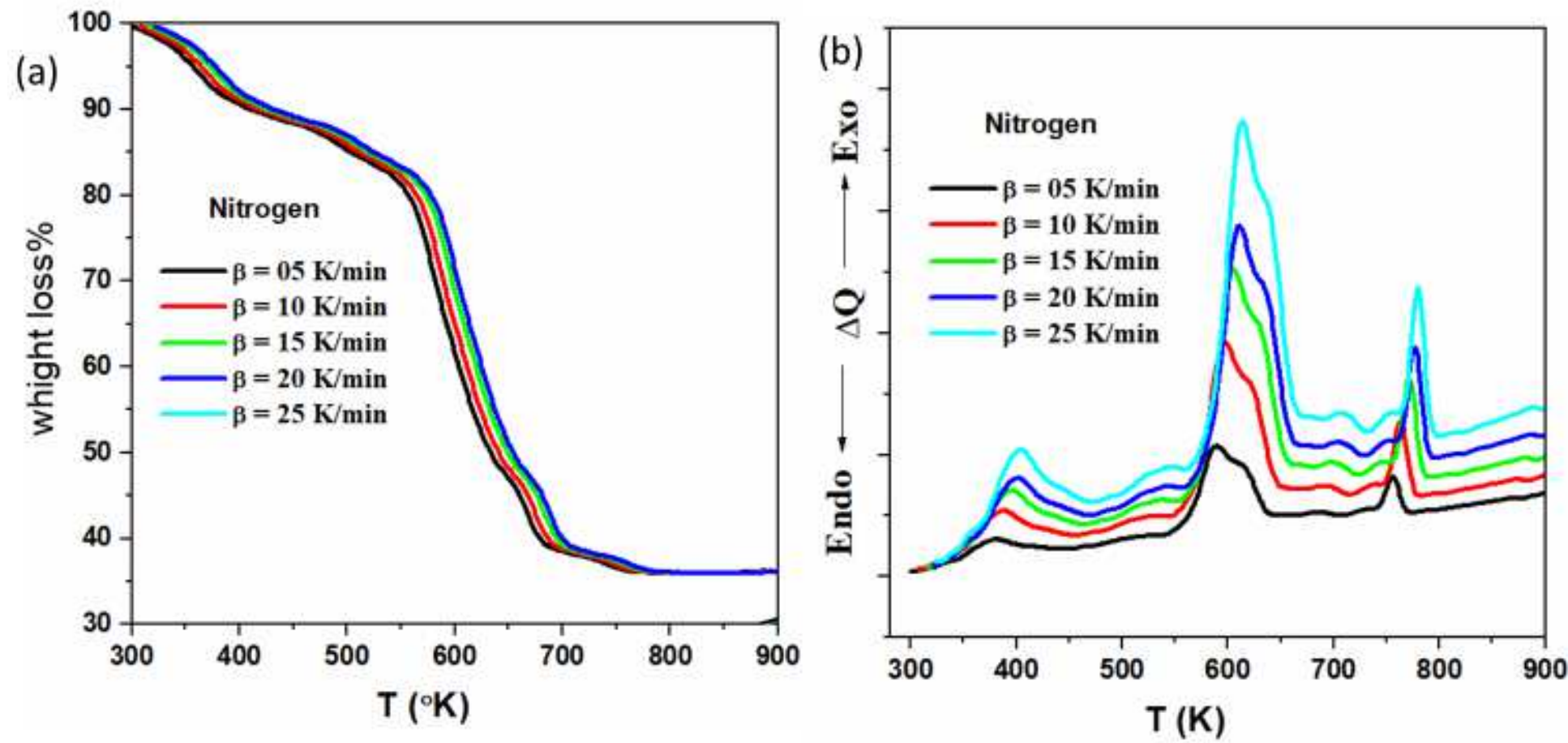

Figure 2

See the Supplemental Files section for the complete figure caption 

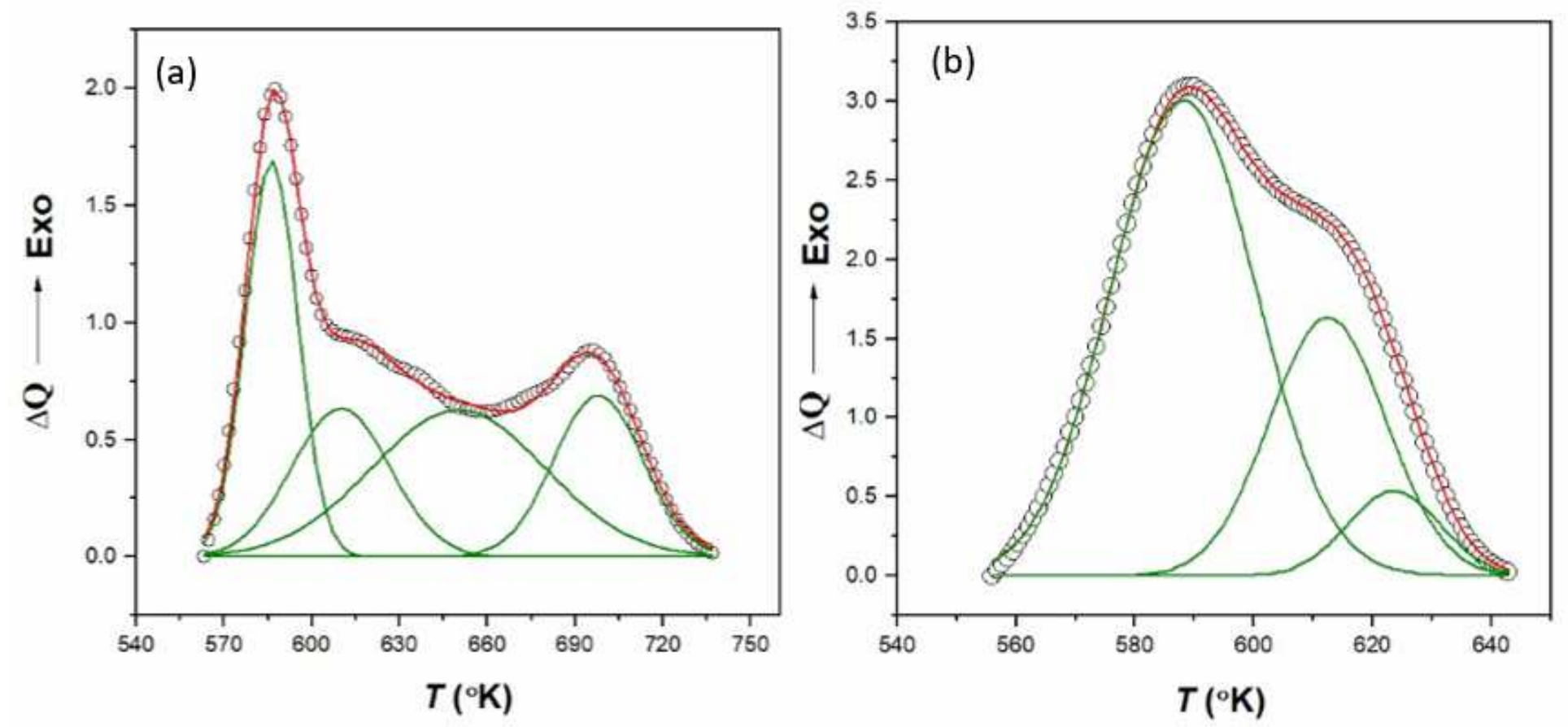

Figure 3

See the Supplemental Files section for the complete figure caption
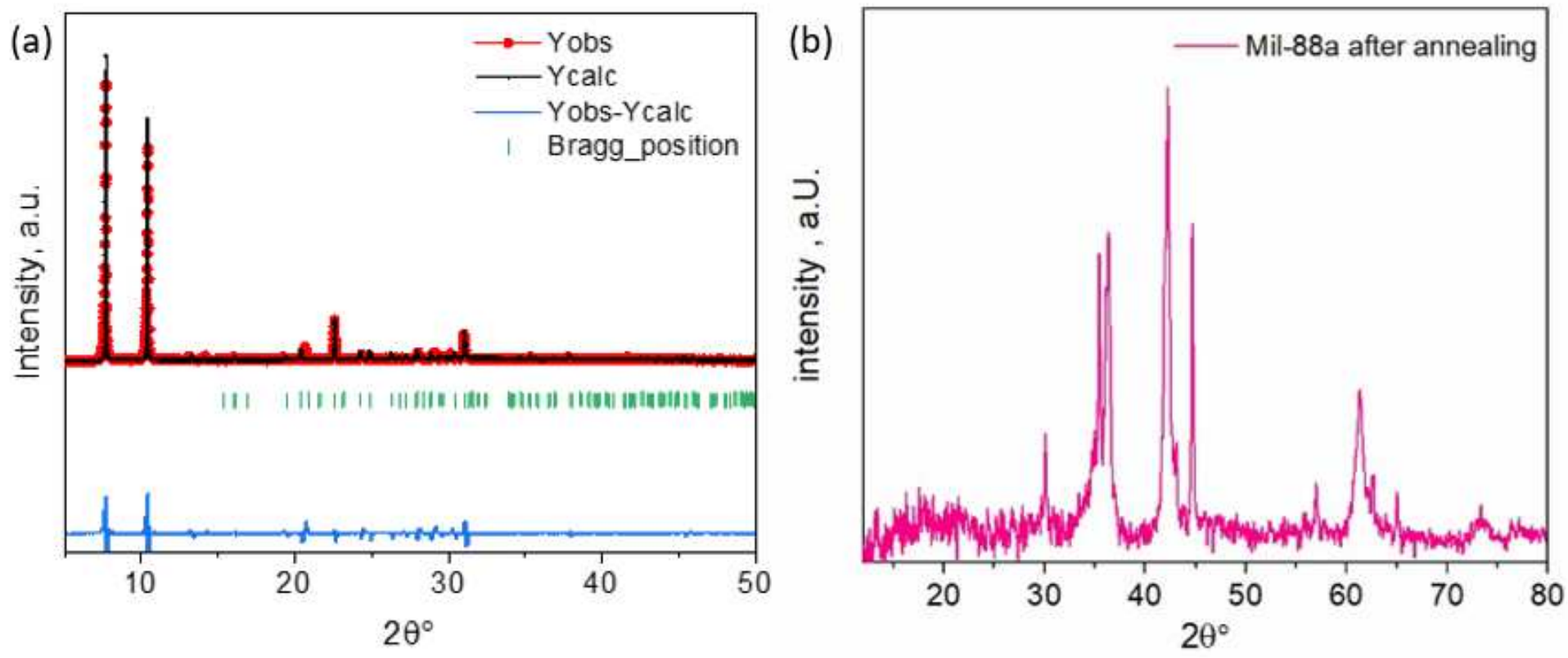

Figure 4

See the Supplemental Files section for the complete figure caption 

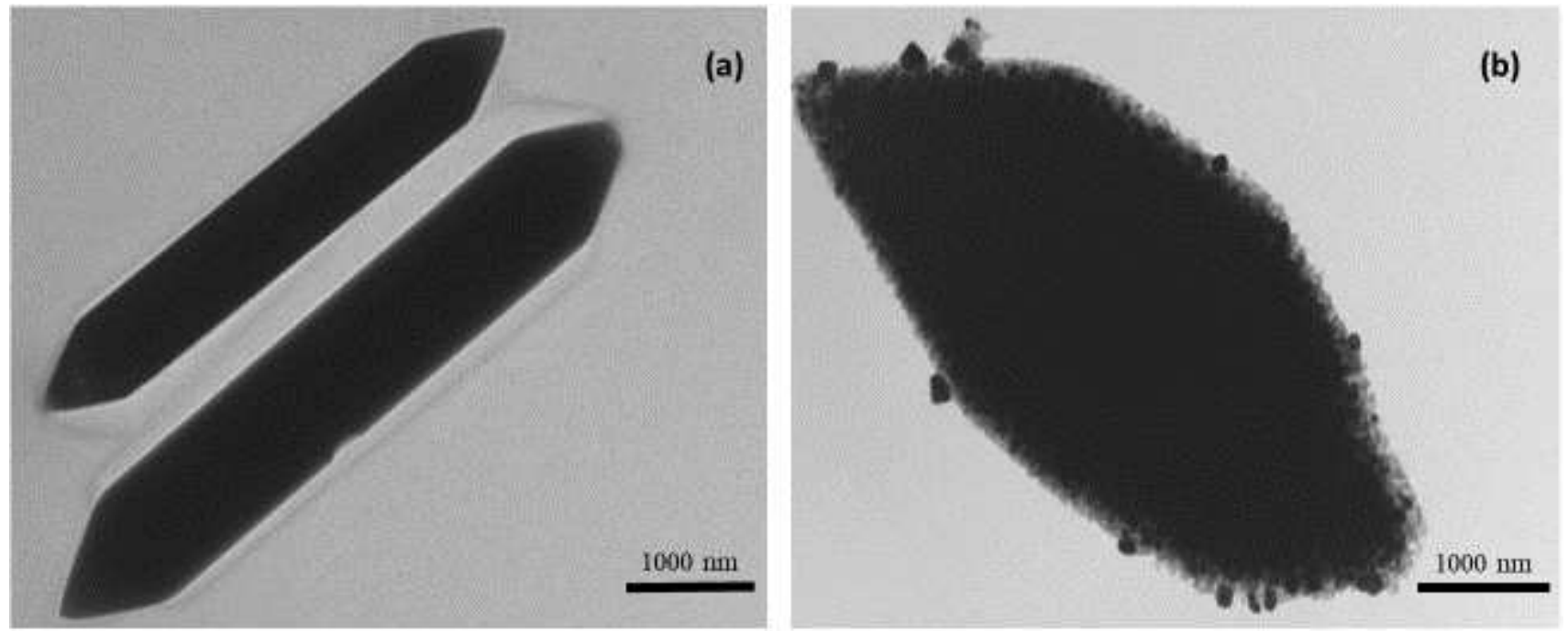

Figure 5

See the Supplemental Files section for the complete figure caption
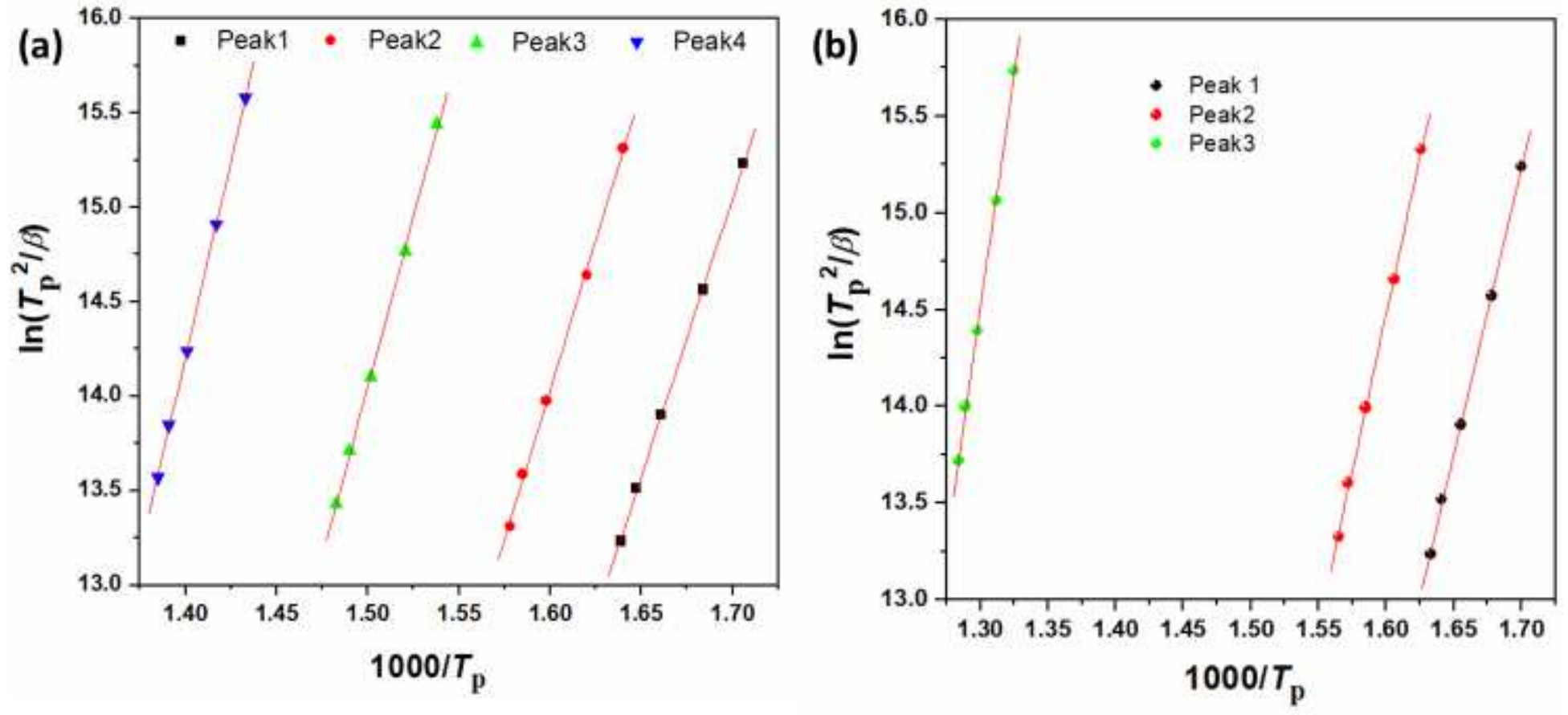

Figure 6

See the Supplemental Files section for the complete figure caption

\section{Supplementary Files}

This is a list of supplementary files associated with this preprint. Click to download.

- FigureCaptions.doc 